\title{
The Head-On Collision of Two Equal Mass Black Holes
}

\author{
Peter Anninos ${ }^{(1)}$, David Hobill ${ }^{(1,3)}$, Edward Seidel ${ }^{(1,2)}$, Larry Smarr ${ }^{(1,2)}$ and Wai-Mo Suen ${ }^{(4)}$ \\ (1) National Center for Supercomputing Applications \\ 605 E. Springfield Ave., Champaign, IL 61820 \\ (2) Department of Physics \\ University of Illinois, Urbana, IL 61801 \\ (3) Department of Physics and Astronomy \\ University of Calgary, Calgary, Alberta, Canada T2N 1N4 \\ (4) Department of Physics \\ Washington University, St. Louis, Missouri, 63130
}

(July 18, 2018)

\begin{abstract}
We study the head-on collision of two equal mass, nonrotating black holes. Various initial configurations are investigated, including holes which are initially surrounded by a common apparent horizon to holes that are separated by about $20 M$, where $M$ is the mass of a single black hole. We have extracted both $\ell=2$ and $\ell=4$ gravitational waveforms resulting from the collision. The normal modes of the final black hole dominate the spectrum in all cases studied. The total energy radiated is computed using several independent methods, and is typically less than $0.002 M$. We also discuss an analytic approach to estimate the total gravitational radiation emitted in the collision by generalizing point particle dynamics to account for the finite size and internal dynamics of the two black holes. The effects of the tidal deformations of the horizons are analysed using the membrane paradigm of black holes. We find excellent agreement between the numerical results and the analytic estimates.
\end{abstract}

PACS numbers: 04.30.+x, 95.30.Sf, 04.25.Dm

\section{INTRODUCTION}

The spiralling coalescence of two black holes in orbit about one another is considered to be one of the most promising sources of gravitational waves [1]. The strong burst of gravitational waves resulting from such an event should be detectable by the next generation of gravitational wave detectors such as LIGO and VIRGO [2]. From observing these violent events in our universe, we expect to obtain important insights into astrophysics, gravitation and cosmology. In particular, such signals should provide the first direct and unambiguous evidence for the existence of black holes if the unique signature of the quasinormal modes [3] are excited. The information gained from the detected waveforms should allow one to reconstruct the astrophysical parameters of the system, such as the masses, spin, and orbital angular momentum and linear momentum of the colliding black holes, and the final black hole. Since LIGO and VIRGO are expected to begin taking data by the end of the decade, it is important to perform accurate calculations of the waveforms emitted during these events. Numerically generated waveform templates will be essential for the analysis of data collected by gravitational wave detectors.

In a series of papers [4 6] we investigate a special case of the black hole coalescence problem, namely the head-on collision of two black holes. On the one hand, the simplifying assumption of a head-on collision reduces the general three dimensional coalescence problem to a two dimensional axisymmetric problem, and is hence much more tractable. On the other hand, a head on collision can be regarded as an approximation to the last nonlinear stage of inspiralling coalescence-the final plunge.

Our work extends and refines the earlier calculations of Dewitt, Cadez, Smarr, and Eppley [7] 12 (henceforth abbreviated as DCSE). Results from that collective body of work suggest that the normal modes of the final black hole resulting from the collision are excited and that the total energy released is typically less than $0.1 \%$ of the mass of the final black hole. However these numerical calculations proved to be very difficult due to inherent coordinate singularities and numerical instabilities that plague the two black hole system. Also, the computer power available at that time did not permit highly resolved evolutions, and they did not have waveform extraction techniques [13] at their disposal for determining gauge-invariant waveforms. For these reasons, DCSE quote their results as uncertain to within a factor of two, for example, in the total radiated energy [12]. It is therefore imperative to revisit this important physical problem with the benefit of more powerful computers and improved analytic and numerical techniques developed over the intervening 15 years to calculate unambiguous waveforms and energy fluxes resulting from the collision. 
Building on the work of DCSE, and more recent work involving distorted single black holes [14 16], many of the numerical problems associated with colliding two black holes have been overcome in the present work. In particular, we have used a hybrid set of coordinates to resolve the axis and saddle point problems encountered by DCSE so that our evolutions are more accurate and more stable. The numerical code we have developed can evolve black holes with initial separation distances between $\sim 4 M$ and $\sim 20 M$, where $M$ is the mass parameter defined to be half the ADM mass of the system. (We note that $M$ is simply a convenient parameter to characterize the coordinates and only approximates the mass of a single black hole in the limit that the holes are widely separated.) We have applied more modern analysis techniques on the numerical data to extract waveforms and compute the total energies emitted. Using gauge-invariant waveform extraction we are now able to determine highly accurate $\ell=2$ waveforms and, for the first time $\ell=4$ waveforms. We also present analytic estimates of the total energy radiated in the collision by taking into account the finite size and internal dynamics of the two black holes. Comparisons of these results to the numerical solutions are valuable not just as a confirmation of our results, but more importantly, they provide a physical understanding of the numerical data.

Sec. II provides an overview of the basic theoretical and computational methods used in this work. A more complete discussion of the initial data, the choices of coordinate systems, gauges, and the numerical methods we developed to handle the problems associated with the evolution of two black hole spacetimes can be found in a companion paper [6]. In Sec. III] we present results from our numerical studies. First we investigate the highly nonlinear and dynamical near field region by looking at the evolutions of the metric data and apparent horizons. We further discuss the timing of the merging of the holes and its implications. Next we turn to the far field and discuss the extraction of gravitational waveforms and compute the total energy radiated from the collision process using various radiation indicators. In Sec. IV, we present a semi-analytic approach to estimate the total energy radiated and compare these results to the numerical calculations obtained in Sec. III. We conclude with Sec. V by summarizing our results and outlining a program to extend this work to more general black hole interactions.

\section{COMPUTATIONAL FRAMEWORK}

We use the 3+1 (ADM) formalism [17 to write the Einstein equations as a first order (in time) set of differential equations for the dynamical variables $\gamma_{i j}$ and $K_{i j}$, the spatial 3-metric and extrinsic curvature respectively. The maximal slicing condition $\operatorname{Tr} K=0$ is imposed throughout the evolution. Also, because the spacetimes we work with possess an axial Killing vector $\left(\partial / \partial x^{3} \equiv \partial / \partial \phi\right)$ all variables are independent of the azimuthal angle $\phi$.

This work, like that of DCSE, is based upon studying the axisymmetric evolution of the analytic Misner initial data [18] representing two equal mass black holes at the moment of time symmetry $\left(K_{i j}=0\right)$. The spatial 3-metric for this data set can be written using cylindrical coordinates as

$$
d l^{2}=\Psi_{M}^{4}\left(d \rho^{2}+d z^{2}+\rho^{2} d \phi^{2}\right),
$$

where

$$
\Psi_{M}=1+\sum_{n=1}^{\infty} \frac{1}{\sinh (n \mu)}\left(\frac{1}{\sqrt{\rho^{2}+\left(z+z_{n}\right)^{2}}}+\frac{1}{\sqrt{\rho^{2}+\left(z-z_{n}\right)^{2}}}\right),
$$

and $z_{n}=\operatorname{coth}(n \mu)$. The free parameter $\mu$ determines the total ADM mass of the spacetime and the proper distance between the two throats. The effect of increasing $\mu$ is to set the two black holes (centered along $\rho=0$, at $z= \pm \operatorname{coth} \mu$ ) further away from one another and to decrease the total mass of the system.

The Misner data consists of two throats connecting two isometric sheets. The throats are spheres on which boundary conditions relating the metric across the two sheets may be imposed. Since the natural boundaries (the throats and a sphere surrounding the system far from the throats) do not lie along constant $(z, \rho)$ coordinates, it is useful to introduce the body-fitted Cadez [7] coordinates $(\eta, \xi)$ where $\eta$ is the logarithmic "radial" coordinate and $\xi$ the "angular" coordinate. The advantage afforded by this set of coordinates (shown in Fig. 1) is that they are spherical near the throats of the black holes and also far away in the wave zone, thus allowing us to deal with throat boundaries and asymptotic wave form extractions in a convenient way. The disadvantage is that the coordinate transformation introduces a singular saddle point at the origin $(z=\rho=0)$ that is not present in cylindrical coordinates. This creates certain numerical difficulties that require special treatment as we discuss briefly in the remainder of this section. The success of our methods depends critically on utilizing both sets of coordinate systems (cylindrical and Cadez) to advantage.

We have investigated a number of different numerical schemes to solve the problem of colliding two black holes head-on. The basic idea that evolved from our investigations is to solve for the Cadez metric components, which we write as 


$$
\gamma_{i j}=\Psi^{4} \hat{\gamma}_{i j}=\Psi^{4}\left(\begin{array}{ccc}
A & C & 0 \\
C & B & 0 \\
0 & 0 & D \sin ^{2} \xi
\end{array}\right)
$$

in the coordinate order $(\eta, \xi, \phi)$, on the Cadez grid and use a shift vector to set $C=\partial_{t} C=0$. (We note that only the conformal metric components are evolved. The conformal factor $\Psi^{4}=\Psi_{M}^{4} / J$, where $J$ is the Jacobian of the Cadez/cylindrical coordinate transformation, remains constant in time.) This choice for the shift vector has the advantage of a diagonal 3-metric which helps to suppress the axis instability and simplifies the equations of evolution and the extraction of invariant gravitational waves in the far field. Furthermore, with this approach it is possible to define variables for the two black hole system that obey the same evolution equations with similar boundary conditions as the single distorted black hole code developed in previous work [14, 15. In fact, the two black hole code in its final incarnation evolved from the code we developed for distorted axisymmetric single black hole spacetimes and much of the discussion in 14,15] is directly applicable here.

The difficulty with Cadez coordinates is the singular saddle point located within the computational domain at the origin $z=\rho=0$ (see Fig. 1). We evolve data near the saddle point by taking advantage of the fact that the spacetime metric components in cylindrical coordinates are smooth everywhere, including the saddle point. We can therefore define a cylindrical coordinate "patch" to evolve the cylindrical metric and extrinsic curvature components on the Cadez grid over regions near the saddle point. The two sets of components, Cadez and cylindrical, are evolved everywhere independently of each other (except for the coupling at the patch boundaries) on a single Cadez grid. The nonsingular cylindrical components are then used to correct the singular Cadez components in the patched region using the general tensor relations $T_{i j}^{\prime}=\left(\partial x^{k} / \partial x^{i}\right)\left(\partial x^{l} / \partial x^{\prime j}\right) T_{k l}$. The Cadez components, in turn, provide corrections to their cylindrical counterparts everywhere else, helping to suppress the axis instability that is inherently present in the cylindrical coordinate system possessing a nondiagonal metric. A more detailed discussion of this procedure can be found in $[6]$.

Our code was subjected to a number of tests, including matching waveforms to perturbation theory as we do in Sec. III B 1. We also performed various convergence studies using 100 (27), 200 (35) and 300 (55) radial (angular) zones. We have shown that the convergence rate for the total radiated energy is quadratic in the grid spacing and, more specifically, differences in the dominant $\ell=2$ waveforms between the 200 and 300 radial zone evolutions is on the order of just a few percent. We discuss the accuracy and reliability of our calculations throughout this article when appropriate and refer the reader to Ref. [6] for more details.

\section{NUMERICAL RESULTS}

We have investigated six separate cases of the Misner two black hole data sets corresponding to different values of $\mu$. The physical attributes of the initial data for these six cases are summarized in Table for where we show $M=M_{A D M} / 2$ (ie., half the ADM mass), the proper distance between the two throats, and whether the data contains a single global apparent or event horizon surrounding the two holes. In this section, we categorically discuss the dynamics of both the near and far field regions for the various cases.

\section{A. Near Field}

1. Spatial Metric

First we present results for the case $\mu=1.2$, which is a data set corresponding to two holes that have already merged initially. The initial data contains an apparent horizon that encircles both throats. In Fig. 2 we show the conformal metric function $\hat{\gamma}_{\eta \eta}=A$ at the coordinate time $t=25 \mathrm{M}$. Notice that a sharp peak surrounding the hole is developing. This peak develops essentially spherically around the two throats from early in the evolution, showing that the system behaves as a single black hole from the outset. These results are similar to those observed in studies of single throat spacetimes 114.15]. The reasons behind the development of the peak is clear: As the coordinates are dragged into the hole, the proper distance between radial grid points increases rapidly towards the throat. However, as shown in Fig. 3 as the lapse goes to zero in the region near the throats, "freezing" all motions there. Hence we see only the growth of the proper distance between grids in coordinate time in the region near the horizon, developing a sharp peak. Such grid stretching effects present one of the main difficulties in evolving black hole spacetimes in any numerical simulation utilizing a singularity avoiding time slicing. During the course of evolution, the dramatic change in the radial metric function becomes increasingly more difficult to resolve numerically, and the region of 
causal dependence eventually becomes much smaller than the coordinate grid spacing (as the radial metric function increases and the lapse decreases there).

For comparison, we show in Fig. 囵 the lapse for the case $\mu=3.25$ where the throats are much more separated, forming two black holes initially. The display corresponds to an early time $(t=22.5 M)$ in the evolution of the system where the two holes are acting essentially independently of each other as they begin to fall together, and the collapse of the lapse locally around the throat reflects this fact. After the holes begin to coalesce, the lapse collapses spherically around both throats which are contained within the final black hole.

\section{Horizons}

We have already observed that at late times the intrinsic geometry of the apparent horizons oscillates at the normal mode frequency of the final black hole. This aspect of apparent horizon dynamics is discussed in detail in Ref. [5] for three different spacetimes including colliding black holes. Here we look at horizons in the context of distinguishing one from two black hole data sets and estimating the mass energy of radiated gravitational waves.

One can define an effective mass of a black hole based on its apparent horizon via the relation 19

$$
M_{h}=\sqrt{\frac{A_{h}}{16 \pi}}
$$

where $A_{h}$ is the intrinsic area of the apparent horizon. This relationship gives a lower limit for the mass of the black hole, since we know the apparent horizon should lie within or (in the case of stationary black holes) coincide with the actual event horizon. When the system begins to settle down Eq. (1) provides a good estimate of the black hole mass as the apparent horizon will lie very close to the event horizon.

In Fig. 1 we show the evolution of the horizon mass computed from Eq. (四) for two different cases. The numerical data for the case $\mu=1.2$ is shown as a solid line. The mass of the hole $M_{h}$ is normalized to units of the total ADM mass of the spacetime, so ideally we should have $M_{h}<1$ for all time. However, because the horizon is always found near the peak of $\hat{\gamma}_{\eta \eta}, M_{h}$ is extremely sensitive to the precise position of the located horizon as discussed, for example, in Ref. [5]. Small errors in the height and shape of the metric functions arising from inadequate resolution can exaggerate errors in the area of the horizon derived from these metric functions. A typical effect of this problem is to overestimate the horizon mass $M_{h}$ after the metric functions become too sharp for the grid to resolve. (This effect has also been discussed in Ref. [20] where an apparent horizon boundary condition was used to circumvent this problem.)

Also shown in Fig. is the result for the case $\mu=2.2$. In this case the initial horizons are the two distinct throats and contain only about $79 \%$ of the total mass of the spacetime. Because we use a slicing condition in which the lapse is zero on both throats, each throat remains a marginally trapped surface throughout the evolution. We track this surface only until another trapped surface forms across the equator $(z=0)$ to surround both throats. This new horizon, when it forms, contains essentially all the mass of the spacetime except for a small amount carried by the radiation. Ideally one should see the horizon mass leveling off just below the total ADM mass of the system, with the difference being accounted for by the energy carried away by gravitational radiation. However the errors in the horizon mass, due to effects discussed above, are large enough to hide this small amount of radiation which is less than $0.1 \%$ of the total mass. Fig. 5 also shows a spurious feature appearing at the time of the formation of the new horizon. This feature is due to numerical difficulties with locking on to the new horizon which first appears near the coordinate singularity present in the Cadez coordinate system. After a brief period, the system settles to the final black hole configuration (apart from the numerically induced slow growth in the horizon mass as discussed above.)

The results for holes with wider initial separations are similar to the case $\mu=2.2$, except that the time scale for the merging of the holes is longer. Thus, by the time the holes have merged, as measured by the appearance of an outer horizon, the horizon mass is overestimated by larger amounts. In the case $\mu=3.0$ this effect is about $20 \%$. Note that the wavelength of the quasinormal mode of a black hole scales as its mass, so this is consistent with the results of Sec. (III B 1) which show that the extracted waveform for these cases have wavelengths slightly longer than expected for a final hole of mass $M_{A D M}$.

Although apparent horizons can confirm when two black holes have definitely merged, the presence of two distinct apparent horizons does not guarantee that the holes are separate, as a global event horizon may still surround the individual holes. The claim that the larger $\mu$ cases represent two distinct black holes is supported by computing the area of a 2-sphere defined on a constant $\eta$ surface that just encircles the saddle point and therefore both holes. In Fig. 6 we plot the area of this surface on the initial time slice as a function of separation (and mass) parameter $\mu$. The horizontal line is $16 \pi$, the area corresponding to a surface representing an effective mass equal to the ADM mass (our 
units are such that the ADM mass is normalized to unity). The point of intersection is $\mu \sim 2.1$, and Fig. 6 indicates that for larger values of $\mu$ the data represents two black holes.

A more convincing and precise argument can be realized by integrating light rays radially outward along the equator starting from midway between the black holes. If the photons "escape", then clearly the system contains two disjoint event horizons initially and hence two separate black holes. (Whether photons escape or not is determined by their position relative to the apparent horizon at late times, typically $\sim 80 M$. At such late times the apparent horizon is expected to lie near the event horizon.) We have performed several such studies in an attempt to find a critical value for $\mu$ that separates the initial data into one or two black hole sets. Our results indicate that this value is approximately 1.8. For $\mu \gtrsim 1.8$ the photons escape, while for $\mu \lesssim 1.8$ they do not. Furthermore, we have recently developed a method for tracing out the actual event horizon surface [21] and found results consistent with those obtained by integrating photons.

\section{Collision Timings}

In Fig. 7 we compare different timings of the collision for each evolution considered. The meaning of the various timings is as follows: $t_{\text {newt }}$ is the Newtonian free fall time for two point particles to collide from rest and separated initially by a distance $L / M, t_{h o r}$ is the time at which the first merged apparent horizon appears, and $t_{\text {ring }}$ is the time at which the first (negative) peak in the $\ell=2$ gauge-invariant waveform reaches the detector at $r=40 M$, indicating the (retarded) onset of the quasinormal ringing of the final black hole. First we note the remarkable coincidence between the free-fall times to collision and the time required for the apparent horizons to merge. This agreement between such different indicators of the coalescence time is very satisfying.

Another interesting feature is the timing of the onset of the quasinormal ringing. Note that for $\mu$ below 2.2 $(L / M<8.9)$ the ringing begins at about the same time regardless of the separation between the holes. For holes separated by more than this, a delay in the onset of ringing becomes apparent. The fact that the ringing begins at the same time regardless of their separation is not surprising for $\mu$ below 1.8. As we discussed above, the two throats are really just a single distorted black hole and the separation between throats is physically irrelevant. For $\mu$ between 1.8 and 2.2, as the two holes are initially disjoint, one might expect a delay in the onset of ringing. However, the important physical property of the system governing the quasinormal ringing is the gravitational scattering potential barrier surrounding the holes, not the position of the event horizon itself. Since the peak of this potential barrier is located near $r=3 M$ for a Schwarzschild hole and the horizon is at $r=2 M$, we expect the potential barrier of each hole to merge into a single one before the horizons do. Therefore, for black holes that are initially close enough that their potential barriers have effectively merged, we expect to see a system that behaves essentially like a single distorted black hole in terms of quasinormal mode ringing, even if the event horizons are distinct. From Fig. 17it is clear that this transition from merged potentials to distinct potentials takes place near $\mu \sim 2.2$.

\section{B. FAR FIELD}

\section{Gauge-Invariant Waveform Extraction}

The main method we use to calculate waveforms is based on the gauge invariant extraction technique developed by Abrahams and Evans [13] and applied in Ref. [16] to black hole spacetimes. The basic idea is to split the spacetime metric into a spherically symmetric (static) background and a small perturbation in the region where the curvature is dominated by the mass content of a small compact object. We first expand the metric perturbation in $m=0$ spherical harmonics $Y_{\ell 0}(\theta)$ and their tensor generalizations. The Regge-Wheeler perturbation functions are then extracted from the numerically computed metric components and used to construct the gauge invariant Zerilli function $\psi$. (See [13] for a detailed discussion of this procedure.) $\psi$ represents the wavelike part of the metric that is radiative at large distances from the source and is commonly used in semi-analytic calculations of black hole normal mode frequencies [3]. The asymptotic energy flux carried by gravitational waves can be computed from

$$
\frac{d E}{d t}=\frac{1}{32 \pi}\left(\frac{\partial \psi}{\partial t}\right)^{2}
$$

independently for each $\ell$ mode contribution for the normalization we use for $\psi$.

For all of the cases studied in this paper we have extracted both the $\ell=2$ and $\ell=4$ waveforms at radii of 30,40,50,60, and 70M. (Coordinate positions corresponding to physical distances in units of $M$ are approximated 
from the initial data in the asymptotically spherical far field as $r \sim \sqrt{\gamma_{\xi \xi}} / M=\Psi^{2} / M$.) By comparing results at each of these radii we are able to check the propagation of waves and the consistency of our energy calculations.

In Fig. $\mathrm{O}$ we show the $\ell=2$ waveform (solid line) extracted at a radius of $40 M$ for the case $\mu=1.2$. This result is similar to waveforms extracted from simulations of single distorted black holes (see, for example, Ref. [16]) even though the initial data sets and coordinate systems used are significantly different. Of course since there is a horizon surrounding both throats, we expect this system to evolve as a single black hole from the outside. As a perturbed single black hole system, we also expect the quasinormal modes of the black hole to be excited. The dotted line in Fig. 8 shows the fit of the lowest two (fundamental and first overtone) $\ell=2$ modes of a black hole of mass $(2 M)$, over the range $70<t / M<160$, obtained from Refs. [22,23]. The fit is excellent, showing that the normal mode is the dominant part of the emitted radiation. We note that the first overtone quasi-normal mode is much more strongly damped than the fundamental, and hence does not contribute appreciably to the fit at late times. Its main effect is to increase the accuracy of the fit to the first peak in the extracted waveform.

Next we discuss the case $\mu=2.2$, for which there are no initial common apparent nor event horizons. In Fig. 9 we show the $\ell=2$ extracted waveform for this case. The solid line shows the waveform detected at a distance $r=40 M$ and the long dashed line shows the waveform extracted at $r=60 \mathrm{M}$. The wave is clearly propagating away from the hole at light speed with essentially invariant shape and amplitude, with a wavelength of $2 \times 16.8 M$, confirming the original findings of Smarr and Eppley [12]. However, our more accurate code now allows us to go beyond estimating the wavelength and to fit quantitatively the waveform to results known from black hole perturbation theory. The short dashed line shows the result of fitting the $r=40 M$ waveform (in the range $64 M<t<160 M$ ) to a linear combination of the fundamental and first overtone of the $\ell=2$ quasinormal mode for the final black hole with mass $2 M$. The fit is quite good, matching both the wavelength and damping time, showing that the final black hole mass is indeed very close to the total mass of the spacetime.

In Fig. 10 we show the more difficult $\ell=4$ waveform for the same case $\mu=2.2$, extracted at the same radius $r=40 M$. Again this waveform has been fit (over a similar range) to a superposition of the fundamental and first overtone $\ell=4$ quasinormal modes of the black hole. Although the fit to the extracted waveform is reasonably good in terms of the wavelength and damping time, this waveform is rather sensitive to the computational parameters such as grid resolution and the extent of the numerical "patch" of cylindrical metric functions covering the saddle point. As discussed in Ref. [6], the amplitude of the $\ell=4$ waveform can vary by about a factor two over a wide range of patch, diffusion, and resolution parameters. Future refinements of this code may allow us to make more definitive predictions of this difficult waveform extraction.

Finally, in Figs. 11 and 12 we show the $\ell=2$ and $\ell=4$ waveforms respectively for the case $\mu=2.7$, where the holes are well separated by about $12.6 \mathrm{M}$ initially. The solid lines are the waveforms extracted at a distance $r=40 M$. In this case the fits to perturbation theory are still reasonably good, but are not as close as the calculations performed for holes that are initially closer together. The wavelengths of the extracted waveforms are somewhat too long, and this can be understood as a numerical artifact of our methods. The calculation must run for a longer period of time before the onset of quasinormal ringing, so the peak in the radial metric function becomes more difficult to resolve. This leads to an error in the longitudinal (spherical) part of the field, that causes the effective gravitational scattering potential to be somewhat different from the true potential. (See also Ref. 16 for a discussion of this point.) Since this potential is critical in determining the quasinormal frequencies of the system, the normal modes are generated at slightly different frequencies.

\section{Other Radiation Indicators}

The Newman-Penrose scalar [24]

$$
\Psi_{4}=R_{\alpha \beta \gamma \delta} l^{\alpha} \bar{m}^{\beta} k^{\gamma} \bar{m}^{\delta}
$$

provides another approach that can be used to treat the problem of radiation extraction. The vectors $k$ and $l$ are orthogonal real vectors defined by adding and subtracting a spacelike unit vector with a unit timelike vector. The vector $m$ and its complex conjugate $\bar{m}$ are orthogonal null vectors tangent to the surface of a 2 -sphere representing the wavefront of an outgoing shell of radiation. The basis set we have chosen to work with is the following

$$
\begin{aligned}
k_{\mu} & =\frac{1}{\sqrt{2}}\left[-\alpha+\Psi^{2} \sqrt{A} \beta^{\eta}, \Psi^{2} \sqrt{A}, 0,0\right], \\
l_{\mu} & =\frac{1}{\sqrt{2}}\left[-\alpha-\Psi^{2} \sqrt{A} \beta^{\eta},-\Psi^{2} \sqrt{A}, 0,0\right],
\end{aligned}
$$




$$
\begin{aligned}
& m_{\mu}=\frac{1}{\sqrt{2}}\left[\Psi^{2} \sqrt{B} \beta^{\xi}, 0, \Psi^{2} \sqrt{B}, i \Psi^{2} \sqrt{D \sin ^{2} \xi}\right] \\
& \bar{m}_{\mu}=\frac{1}{\sqrt{2}}\left[\Psi^{2} \sqrt{B} \beta^{\xi}, 0, \Psi^{2} \sqrt{B},-i \Psi^{2} \sqrt{D \sin ^{2} \xi}\right] .
\end{aligned}
$$

Far from the source $\Psi_{4}$ represents an outward propagating wave and is therefore naturally normal to a 2 -sphere of constant "radius" $\eta$. The total radiated energy loss can therefore be estimated by 25]

$$
\frac{d E}{d t}=\frac{1}{4 \pi} \oint\left[\int_{0}^{t} d t^{\prime} \Psi_{4}\right]^{2} r^{2} d \Omega
$$

where the integration is over a 2-sphere (with an area of $4 \pi r^{2}$ and a surface element $d \Omega$, where $r \sim \sqrt{\gamma_{\xi \xi}} / M=$ $\left.\Psi^{2} \sqrt{B} / M\right)$ lying in a spacelike hypersurface surrounding the radiating system.

A third method that we have used to track gravitational radiation is based on the Bel-Robinson vector [26]

$$
p^{\gamma}=E_{\alpha \beta} \epsilon^{\beta \gamma \delta} B_{\delta}^{\alpha} .
$$

where $E_{\alpha \beta}$ and $B_{\alpha \beta}$ are the "electric" and "magnetic" components of the four dimensional Riemann tensor. $\varepsilon_{\alpha}^{\gamma \mu}=\varepsilon_{\alpha \beta}^{\gamma \mu} n^{\beta}$ is the 3-dimensional Levi-Civita permutation tensor and $n^{\alpha}$ is the unit vector normal to the spacelike $t=$ constant hypersurfaces. Although $p^{\gamma}$ is constructed in a manner formally similar to the Poynting vector of electromagnetism, we note that it is not a physical momentum vector for gravitational waves, since its units differ by $M^{2}$. Nevertheless, $p^{\gamma}$ has been proven to be effective in qualitatively tracking gravitational radiation [11,27], and as demonstrated by DCSE in Ref. [12] and again in section [II B3, the radiated energies computed using $p^{\gamma}$ are in good quantitative agreement with those computed using other radiation indicators. Since $p^{\gamma}$ is dimensionally a flux vector quantity, the energy loss can be approximated by integrating the radial energy flux $p^{r}$ over a closed 2-sphere as described above for $\Psi_{4}$

$$
\frac{d E}{d t}=\frac{1}{4 \pi} \oint\left[\int_{0}^{t} d t^{\prime}\left( \pm \sqrt{\left|p^{\gamma} r_{\gamma}\right| / 2}\right)\right]^{2} r^{2} d \Omega .
$$

The choice of sign in the integral of (13) is taken to be the sign of $\Psi_{4}$. Equation $(13)$ is motivated by the asymptotic form $p^{\gamma} r_{\gamma} \rightarrow 2\left|\Psi_{4}\right|^{2}$ applicable to the case of monochromatic waves in linearized Minkowski spacetimes [11]. This construction yields results that are consistent with the integrals of $\psi$ and $\Psi_{4}$ in the asymptotic far field.

It is informative to compare the waveforms obtained by the three different methods. In Fig. 13, we show the $\ell=2$ Zerilli function $\psi$ (solid line), $\Psi_{4}$ (dashed line) and $p^{r}$ (dotted line) at a fixed point $r=70 M$ along the equator for the case $\mu=2.2 . \Psi_{4}$ and $p^{r}$ have been normalized to the same scale of $\psi$ by matching the amplitudes of their maximum peaks. The $\ell=2$ fundamental quasinormal mode is clearly present and dominant in all three signals.

\section{Energies Radiated}

The total radiated energy $E$ can be computed from the Zerilli function using Eq. (5). We display these results in Fig. 14. The six clusters of unconnected symbols represent the six numerical simulations corresponding to the different $\mu$ parameter values. Each of the five symbols within a cluster corresponds uniquely to the total integrated $\ell=2$ energy computed at the five different wave detectors. For reference, the early results of Smarr and Eppley are plotted as large crosses with error bars suggested by Smarr [12]. Within the large errors quoted, those early results are remarkably consistent with our more accurate results.

Clearly the results in Fig. 14 show two distinct regimes, as denoted by the arrows in the upper part of the figure. For $\mu<1.8$ the initial data contains one black hole, as discussed in section III A 2, and the energy radiated falls off exponentially. We have fit the energy output to an exponential for $\mu \leq 1.8$ and find that the results are approximated well by the formula $E=3.13 \times 10^{-8} \exp (4.852 \mu)$. For $\mu>1.8$ there are two holes and the energy radiated is somewhat independent of the initial separation. Three lines based on analytic and semi-analytic calculations that treat the system as two black holes are also shown, as we discuss in more detail in the next section. The curve labelled "DRPP calculation" is based on Ref. [28], the result labelled "Reduced Mass Correction" takes into account the finite mass of a black hole falling into its partner, and the dashed line is the complete semi-analytic calculation discussed in Sec. IV, accounting for other effects. Finally we note that the energy radiated is very small compared with the upper limits based on the horizon area theorem (represented by connected circles in Fig. 14), as discussed in the next section. 
Table II compares the total radiated energy computed using three different radiation variables: the Zerilli function $\psi$, the Newman-Penrose scalar $\Psi_{4}$ and the Bel-Robinson vector $p^{r}$. The energies in Table II are results from simulations resolved with a $200 \times 35$ grid and are normalized to the total ADM mass of the spacetime $(2 M)$. The range of values quoted for the numerical calculations for each of the six cases represent the range of data across the different detector locations, with the lower (upper) limits corresponding to the outer (inner) detectors. As a whole, the results are remarkably consistent. The various numerical constructions differ significantly only at the innermost detectors for the low $\mu$ cases. These deviations are attributed to several effects. First, the near zones are characterized by stronger highly distorted behavior than the asymptotic wave zones, making this region more difficult to resolve accurately. $\Psi_{4}$ and $p^{r}$ are curvature type variables that have an explicit dependence on first and second order gradients of the metric components. Hence, they are more susceptible to numerical inaccuracies than the Zerilli function which depends only on first derivatives of the 3-metric. Secondly, the curvature quantities are projected onto a coordinate based tetrad, and the Cadez coordinates are distorted from sphericity in the inner regions of the grid, obscuring the physical interpretation of these quantities.

\section{ANALYTIC ESTIMATE}

To gain a physical understanding (and confirmation) of the numerical results, we outline a procedure to estimate analytically the total radiated energy. Our approach is based on the well-studied problem of a test point particle originally at rest at infinity plunging into a Schwarzschild black hole [29, 28, 30 32]. For the test point particle problem, [29] combined a Newtonian quadrupole moment calculation with the linearized theory of Landau-Lifshitz [33] to find the in-flight radiation

$$
E=\frac{1}{105}\left(\frac{m^{2}}{M}\right)
$$

for infall from $\infty$ to $r=2 M$, where $m$ is the mass of the test point particle and $M$ the mass of the black hole with $m \ll M$. In [28] the total radiation for the same test point particle problem is obtained using black hole perturbation theory (see e.g., [3] and references therein). The result is comparable [28]

$$
E=0.0104 \frac{m^{2}}{M} .
$$

Our approach is to adopt the general relativistic result (15) and modify it to include correction factors so that it can describe the two black hole collision. In the following, we shall discuss correction factors due to (A) $m$ is not much smaller than $M$, (B) the infall is not from infinity, and (C) the black hole, unlike a point particle, has a finite size and internal dynamics.

\section{A. mass scaling}

Before we go into the various correction factors, it is useful to understand why $E$ in (15) is proportional to $m^{2} / M$. For $m \ll M$, the quadrupole moment of the system is $I \sim m r^{2} \quad$ where $r$ is the radial distance between $m$ and $M$. The gravitational wave luminosity is given by $\dddot{I}$, the third time derivative of $I$. In the Newtonian approximation, $\dot{r} \sim \sqrt{2 M / r}$ and $\ddot{r} \sim M / r^{2}$, we have

$$
L \propto \dddot{I}^{2} \sim m^{2}(\ddot{r} \dot{r})^{2} \sim m^{2}\left(\frac{M^{3}}{r^{5}}\right) .
$$

The total energy radiated is

$$
E=\int L d t \approx L_{\text {strong field }} \times \delta t_{\text {strong field }} .
$$

The integral is evaluated at the strong field region, as most energy is released towards the end when $m$ is falling near the horizon of $M$. Putting $r=2 M$ into (16) for $L$ in the strong field region, and $\delta t_{\text {strong field }} \approx M$, we have

$$
E \propto \frac{m^{2}}{M},
$$


as in (15).

When $m$ is not much smaller than $M$, it is more accurate to use

$$
I \sim \mu r^{2}
$$

where $\mu \equiv(m M) /(M+m)$ is the reduced mass of the system. Hence the considerations above (equations (16) to (18)) suggest that Eq. (15) should be changed to

$$
E=0.0104 \frac{\mu^{2}}{M}
$$

as the gravitational wave energy output for the case when $m$ is not necessarily much smaller than $M$. Notice that for $m=M$, the $\mu^{2}$ of Eq. (20) introduces a quite significant factor of $1 / 4$.

\section{B. finite infall}

The simple quadrupole approximation of (16) also suggests how the expression should be modified when the infall is not initially from infinity. Starting from rest at a finite distance $r_{o}$ reduces the velocity $\dot{r}$, which enters (16) as $\dot{r}^{2}$. Denote the energy radiated in such a case as $E_{r_{o}}$,

$$
E_{r_{o}}=\int_{t_{o}} L d t=\int_{r_{o}}^{2 M}(L / \dot{r}) d r
$$

Eq. (20) is hence modified to be

$$
\begin{gathered}
E=F_{r_{o}} \times 0.0104 \frac{\mu^{2}}{M} \\
F_{r_{o}}=\frac{E_{r_{o}}}{E_{\infty}}=\frac{\int_{r_{o}}^{2 M} \dot{r}(\ddot{r})^{2} d r}{\int_{\infty}^{2 M} \dot{r}(\ddot{r})^{2} d r}
\end{gathered}
$$

with

$$
\dot{r}=\frac{\left(1-\frac{2 M}{r}\right) \sqrt{\frac{2 M}{r}-\frac{2 M}{r_{o}}}}{\sqrt{\frac{1-2 M}{r_{o}}}} .
$$

Since $r_{o}$ can be as small as a few $M$ in the numerical simulation, we must use the relativistic expression for $\dot{r}$ in Schwarzschild coordinates in Eq. (24). Equations (23) and (24) represent one way of extending the quadrupole formula to the highly relativistic regime. There is no unique way to do the extension, we have just picked a way convenient for our present purpose. This correction factor $F_{r_{o}}$ represents two effects: $(i)$ there is less time to radiate when falling from a finite distance, and $(i i)$ the infalling velocity is smaller. The latter effect is much more important. In Fig. 15 we plot $F_{r_{o}}$ vs. $r_{o}$, the initial Schwarzschild coordinate of $m$, covering the range of $r_{o}$ used in the numerical simulation.

\section{C. internal dynamics}

All considerations up to this point are the same, independent of whether the infalling object is a point particle or a black hole. In the following, we consider correction factors due to this difference. Although at the end we will extrapolate to two black holes of equal mass $m=M$, for both generality and convenience of discussion, we think of the situation as a hole with mass $m$ falling into a hole with $M \geq m$.

As far as the gravitational wave output is concerned, the most important difference between a point mass and a black hole is that a black hole has internal dynamics. There are more channels that the initial gravitational potential energy in the system can dissipate into. Such dissipations decrease the kinetic energy and hence the velocity of the infalling hole. Hence fewer gravitational waves are generated. There are various mechanisms causing dissipation,

which we shall describe separately. Of course, as these dissipative effects are more pronounced in the nonlinear regime near the final coalescence, the separation between the various mechanisms is inevitably of an approximate nature. 


\section{1. tidal heating}

The first kind of dissipation we consider originates from the tidal deformation of $m$ as the hole $m$ falls in the static gravitational field generated by $M$. In the membrane paradigm [34] of black holes, in which the horizon is treated as a 2-D surface living in a 3-D space, endowed with physical properties like viscosity, this tidal deformation heats up the horizon. The heating is described by the horizon equations 34 36

$$
\begin{gathered}
-\frac{d}{d t} \sigma_{a b}+(g-\theta) \sigma_{a b}+\left(2 \sigma_{a c}+\gamma_{a c} \theta\right) \sigma_{b}^{c}=\epsilon_{a b} \\
-\frac{d}{d t} \theta+g \theta-\frac{1}{2} \theta^{2}=\sigma_{a b} \sigma^{a b} \\
\frac{d}{d t} \gamma_{a b}=2 \sigma_{a b}+\gamma_{a b} \theta .
\end{gathered}
$$

Here $\gamma_{a b}$ is the 2-D metric of the horizon of the infalling hole $m, g=1 /(4 m)$ is the surface gravity of the hole $m$, and $\epsilon_{a b}$ is the normalized electric part of the Weyl tensor $\left(C_{a \mu b \nu} l^{\mu} l^{\nu}\right.$, with $l^{\mu}$ the horizon generators). $\theta$ is the expansion rate of the horizon generators

$$
\theta=\frac{1}{\Delta A} \frac{d}{d t} \Delta A
$$

and $\sigma_{a b}$ is the shear of the horizon generators

$$
\frac{d}{d t}(\Delta s)^{2}=2\left(\sigma_{a b}+\frac{1}{2} \theta \gamma_{a b}\right) \Delta x^{a} \Delta x^{b},
$$

with $\Delta x^{a}$ the coordinate separation of the horizon generators.

For a hole with mass $m$ falling in the external tidal field $M / r^{3}, \epsilon_{a b}$ in an orthonormal basis can be approximated by [34]

$$
\epsilon_{\hat{\theta} \hat{\theta}}=-\epsilon_{\hat{\phi} \hat{\phi}} \sim g V \frac{M}{r^{3}}
$$

where $V$ is the velocity of the fiducial observers on the horizon of $m$ moving in the external tidal field $(V=\sqrt{2 M / r}$ for infall from $\infty$ ). Since $\sigma_{a b}$ and $\theta$ in Eqs. (25) 27) are driven by $\epsilon_{\hat{a} \hat{b}}$, which is small in our case, all nonlinear terms in Eqs. (25.27) can be dropped, and $\gamma_{a b}$ is decoupled. $\theta$ is approximately given by 1

$$
\begin{aligned}
\theta & =\int\left[\sigma_{a b}\left(t^{\prime}\right) \sigma^{a b}\left(t^{\prime}\right) G\left(t, t^{\prime}\right)\right] d t^{\prime} \\
& =\int_{r_{o}}^{2 M}\left[\sigma_{a b} \sigma^{a b} G / \dot{r}\right] d r \\
\sigma_{a b} & =\int \epsilon_{a b}\left(t^{\prime}\right) G\left(t, t^{\prime}\right) d t \\
& =\int_{r_{o}}^{2 M}\left[\epsilon_{a b} G / \dot{r}\right] d r
\end{aligned}
$$

where $G\left(t, t^{\prime}\right)$ is the teleological Green's function [34]

$$
G\left(t, t^{\prime}\right)=\left\{\begin{array}{l}
\exp \left[g\left(t-t^{\prime}\right)\right] \text { for } t<t^{\prime} \\
0 \quad \text { for } t>t^{\prime}
\end{array}\right.
$$

\footnotetext{
${ }^{1} \theta$ is infinite where caustics exist on the horizon, although its contribution to the total increase of the horizon area is finite. We do not consider the effect of caustics in this paper.
} 
In Eqs. (31) and (32), $\dot{r}$ is given by Eq. (24); the integrands are regarded as functions of $r$, with $t=t(r)$ obtained by integrating Eq. (24). In Eqs. (31) and (32) the integrations are cut off when the hole falls through the horizon of $M$. In principle the integration should be carried over all times, but the residue is unimportant for the present purpose. In Fig. 16, we plot $\sigma_{\hat{\theta} \hat{\theta}}$ (solid line) and $\theta$ (dotted line) as functions of the Schwarzschild coordinate $r$, for the case of $m=M$. The horizontal and vertical scales are in terms of $M=m=1$.

A fraction of the initial gravitational potential energy $f_{h}=\Delta m / m$ is dissipated into the heating of the horizon of $m$ by this effect:

$$
\begin{aligned}
f_{h}=\frac{\Delta m}{m} & =\frac{1}{2} \frac{\Delta A}{A}=\frac{1}{2} \int \theta d t \\
& =\frac{1}{2} \int_{r_{o}}^{2 m+2 M}[\theta / \dot{r}] d r
\end{aligned}
$$

Notice that the integration in Eq. (34) is terminated at the point when the two holes are engulfed by a common horizon. It is irrelevant whether the object that has fallen in is a point mass or a black hole after that point. We approximated that point to be when the holes are separated by $2 m+2 M$ in Schwarzschild coordinates, i.e., when the two holes are nearly touching. For $m=M$, the heating on the horizon of $M$ is the same as that on $m$, hence the total fraction of energy going into heating of the horizons as the holes are falling in each others tidal field is given by $2 f_{h}$. In Fig. 15, the reduction factor for the energy available for wave generation,

$$
F_{h}=1-2 f_{h}
$$

is plotted against $r_{o}$, the initial separation of the holes, for the case of $m=M$. We see that $F_{h}$ is decreasing with increasing initial separation, as a larger initial separation leads to a larger velocity and in turn larger $\epsilon_{\hat{a} \hat{b}}$ in Eq. (30). As $r_{o} \rightarrow \infty, F_{h}$ decreases to 0.86 . For the range of $r_{o}$ covered in the numerical simulation, this effect reduces the gravitational wave output by about $10 \%$.

\section{2. absorption of gravitational waves}

The second kind of dissipation arises from the fact that, unlike a point mass, a black hole has finite size. As it sweeps through the spacetime, it can reabsorb the gravitational wave already generated in the spacetime.

The gravitational wave that a black hole can absorb depends on the frequency $\omega$ of the wave and the $l-$ pole of the wave:

$$
\text { wave absorbed }=\sum_{l} \int(l-\text { pole wave incident on } m) \times T_{l}(\omega) d \omega \text {. }
$$

Here $T_{l}$ is the transmission coefficient for incident $l$ pole waves as calculated in black hole perturbation theory, see e.g., [2]. We take the $l$-pole wave incident on the infalling black hole $m$ as the $l$-pole wave in the spacetime times the cross-section of the hole $m$, as seen from the hole $M$, i.e.,

$$
\text { cross section } \lesssim \frac{\pi(2 m)^{2}}{4 \pi(2 m+2 M)^{2}}=\frac{1}{4} \frac{\mu^{2}}{M^{2}}
$$

The energy in the $l$-pole wave in the spacetime is given e.g., in Ref. [1], and is reproduced in Fig. 17 (dashed line) for $l=2$ as a function of $\omega$. The vertical axis (for the dashed line) is $d E / d \omega$, all in scale of $M=1$. The transmission coefficient $T_{l=2}$ times 0.1 is also given as a function of $\omega$ (dotted line). Notice that the quasi-normal frequency is $0.3737 m^{-1} . T_{l=2}$ is about 0.5 at this point, dropping to zero rapidly for a smaller $\omega$. As the peak of $d E / d \omega$ is at a smaller $\omega$, the product of $T d E / d \omega$ is small, given by the solid line in Fig. 17 for the case of $m=M$. The area under the curve is found to be $\sim 0.0012 \mathrm{~m}$. Comparing to the total gravitational wave energy in the $l=2$ mode [28], $E_{l=2}=0.0092 m$, Eqs. (36) and (37) lead to about $1 \%$ reabsorption of the $l=2$ wave energy.

Reabsorption of higher $l$ modes can be estimated similarly. However, since the peak of $d E_{l} / d \omega$ is always at an $\omega$ less than the corresponding quasi-normal frequency $\omega_{l}$, whereas the transmission coefficients rise to larger than 0.5 only for $\omega>\omega_{l}$, the reabsorption is always a small fraction of the corresponding component. As the $l=2$ mode is 
the dominant component making $90 \%$ of the total radiation, we see that this reabsorption effect can only lower the total energy output at a $1 \%$ level 2

$$
F_{\mathrm{abs}} \approx 99 \% \text {. }
$$

We point out that there are other mechanisms causing the heating of the black hole horizons during the coalescence of black holes. Indeed, some of them are much larger than the effects considered above. For example, in the late stages of the coalescence, the infalling hole $m$ is moving relativistically and is beaming gravitational waves in the forward direction. Such beaming causes large horizon heating [35,36]. The energy dissipated is an order of magnitude larger than the total gravitational wave output to infinity. However, such an effect is already implicitly included in the result of [28]. Hence no modification factor is needed to account for the process. The reduction in gravitational wave output due to the interference from the different parts of a body for a finite size object falling into a black hole has been estimated in Ref. [37]. For the case of a two black hole collision, the effect is negligible.

\section{D. comparison with numerical results}

In Fig. 14 we plot the final result of the analytic estimate for total gravitational wave energy output

$$
E=F_{r_{o}} F_{h} F_{\text {abs }} \times 0.0104 \frac{\mu^{2}}{M}
$$

versus the initial separation between the two holes, for the case of $m=M$. It is represented by the dashed line. For comparison to the final result of Eq. (39) we have also plotted two intermediate results as straight, solid lines. A simple application of the standard perturbative "DRPP calculation" (Eq. 15) overestimates the energy by roughly an order of magnitude. Replacing $m$ by the reduced mass $\mu$ as in Eq. (20) gives the "Reduced Mass Calculation", accounting for the finite mass of the "perturbing" black hole. The three correction factors $F_{r_{o}}, F_{h}$, and $F_{\text {abs }}$ together act to reduce the energy output further, leading to the curved, dashed line in Fig. 14. In view of the various approximations one has to make to obtain Eq. (39), the agreement between the analytic and the numerical results is remarkable, as the analytic results were obtained without prior knowledge of the numerical results, and vice versa. For $L / M$ less than about 9 the analytic formula overestimates the actual energy output computed numerically. This is to be expected since for small enough separations the holes are initially engulfed by a common effective potential (in the sense of the potential in the black hole perturbation theory), or even a common event horizon. The approximation for colliding black holes in these cases is inappropriate.

The connected circles show the maximum possible radiation output obtained by comparing the initial black hole masses estimated by the areas of the horizons (or a single horizon if the holes are close enough) to the total mass of the spacetime. For large separations this number approaches $29 \%$ as expected from the work of Hawking [19].

What physical understanding is gained from this semi-analytic exercise? The central message is that, as far as gravitational radiation is concerned, a black hole falling into another black hole is not much different from that of a point particle falling into a black hole. As the leading order approximation, the energy output can be described quite well by Eq. 22, which is the test point particle result obtained in 28] modified by insight from the quadrupole formula. We found that the biggest effect due to black hole internal dynamics can be understood as the deformation of the horizons when the holes are falling in the tidal fields of one another. We see that the energy of about $10^{-3} \mathrm{M}$ (for black holes of mass $M$ ) is dissipated by the viscosity of the horizon. Instead of radiating out to infinity, this portion of the initial potential energy is dissipated into the holes, increasing the horizon area. The smallness of this number again testifies that the horizon of a black hole is "stiff", making a black hole rather like a point particle. At present we can only say the contribution from horizon heating is consistent with our numerical results to the level of accuracy. A future direct confirmation of this effect, especially for the case of non-equal mass black holes, will be particularly interesting.

There are other effects that we considered, for example the reabsorption of radiation (also interference calculated in [37]) due to the finite size of the black hole. We found these effects to be negligible beyond the present level of accuracy, adding further weight to the understanding of a black hole behaving to a large extent as a point particle.

\footnotetext{
${ }^{2}$ As the effect of reabsorption is small, various refinements of equations (36) and (37) are not meaningful, e.g., the crosssection (37) is in fact a function of the separation between the two holes and the $\ell=2$ mode of the two black hole system is different from the $\ell=2$ mode of the infalling hole of mass $m$.
} 
There are also other effects that we have thought about but do not know yet how to calculate analytically, for example the increase of the horizon area of the black hole due to caustics. However, the agreement between the numerical results and our present semi-analytic approximations suggests that these other effects most likely do not affect the total energy radiated significantly.

\section{CONCLUSIONS}

We have performed numerical and analytic calculations predicting the gravitational waveforms generated and total gravitational wave energy emitted when two equal mass black holes collide head-on. Waveforms for all cases studied show similar behavior: the normal modes of the final black hole are excited and account for most of the emitted signal. Both the $\ell=2$ and $\ell=4$ waveforms are fit nicely by a superposition of the fundamental and first overtone of the black hole quasinormal modes. Although the fit to perturbative calculations of the $\ell=4$ waveform is quite good, the amplitude and precursor of this waveform are more sensitive to the various computational parameters than the more dominant $\ell=2$ waveform.

The total energy radiated is on the order of $0.002 M$ (where $M$ is half the ADM mass of the spacetime), far below the estimate given by a simple application of the area theorem. The analytic study, appropriate for holes that are initially separate, confirms and elucidates the numerical results. We find the total energy radiated can be approximated quite well using the point particle result modified slightly to account for mass scaling, finite initial separation and internal dynamics of the black holes. Taken together, the analytic and numerical results indicate that even for holes that are initially infinitely separated, the total energy output will be the same order of magnitude. For throats that are close together, Price and Pullin 38] have treated the evolution via gauge-invariant perturbation methods, regarding the system as a single, perturbed hole. They find remarkable agreement with our work for the $\ell=2$ waveforms and total energy radiated, independently confirming our results and providing a new method for evolving distorted black hole data sets. A detailed comparison of the numerical results presented here to semi-analytic results based on regimes where the throats are initially near or far from each other will be published elsewhere.

The work presented in this paper is only a first step on the long path to computing the fully general evolution of three-dimensional, spiralling, coalescing black holes. We expect the more general case to be significantly more complex to compute. In axisymmetry our current calculations can be extended to include: boosted black hole collisions in which the holes are given initial finite velocities, unequal mass black holes which can radiate not only gravitational waves but net linear momentum, and spinning colliding black holes where one can expect more energy to be radiated, particularly if the holes have opposite spin vectors. We intend to pursue such extensions to our present axisymmetric code and to develop and apply new, more general, three dimensional codes [39] to these systems as well.

Finally, we note that we have prepared a video showing results for several of the simulations reported here. Interested readers may contact NCSA media services at the internet address media@ncsa.uiuc.edu for information on how to obtain a copy of the video entitled "The Collision of Two Black Holes." At this address one can also obtain a copy of a videotape of the original movies based on the work of Smarr and Eppley. The NCSA group has also set up a World Wide Web server accessible at the URL http://jean-luc.ncsa.uiuc.edu, and there one can find images and movies of the simulations presented in this paper that cannot be published in traditional form.

\section{ACKNOWLEDGMENTS}

We would like to thank David Bernstein for a number of helpful discussions, Joe Libson and Paul Walker for integrating light rays through the spacetimes, Richard Price and Jorge Pullin for informing us of their results prior to publication, Mark Bajuk for his work on visualizations of our numerical simulations that aided greatly in their interpretation, and Joan Massó for help with preparing some of the graphs for this paper. This work was supported by NCSA, NSF Grant 91-16682, and NSERC Grant No. OGP-121857, and calculations were performed at NCSA and the Pittsburgh Supercomputing Center.

[1] K. Thorne, in Proceedings of the Eight Nishinomiya-Yukawa Symposium on Relativistic Cosmology, edited by M. Sasaki (Universal Academy Press, Japan 1994).

[2] A. A. Abramovici et al., Science 256, 325 (1992). 
[3] S. Chandrasekhar, The Mathematical Theory of Black Holes (Oxford U. Press, Oxford, U.K., 1983).

[4] P. Anninos, D. Hobill, E. Seidel, L. Smarr, and W.-M. Suen, Phys. Rev. Lett. 71, 2851 (1993).

[5] P. Anninos, D. Bernstein, S. Brandt, D. Hobill, E. Seidel, and L. Smarr, Physical Review D (1994), in press.

[6] P. Anninos, D. Hobill, E. Seidel, L. Smarr, and W.-M. Suen, Technical Report, National Center for Supercomputing Applications.

[7] A. Čadež, Ph.D. thesis, University of North Carolina at Chapel Hill, 1971.

[8] L. Smarr, Ph.D. thesis, University of Texas, Austin, 1975.

[9] K. Eppley, Ph.D. thesis, Princeton University, 1975.

[10] L. Smarr, A. Čadež, B. DeWitt, and K. Eppley, Physical Review D 14, 2443 (1976).

[11] L. Smarr, Ann. N. Y. Acad. Sci. 302, 569 (1977).

[12] L. Smarr, in Sources of Gravitational Radiation, edited by L. Smarr (Cambridge University Press, Cambridge, 1979), p. 245.

[13] A. Abrahams and C. Evans, Physical Review D 42, 2585 (1990).

[14] P. Anninos, D. Bernstein, D. Hobill, E. Seidel, L. Smarr, and J. Towns, in Computational Astrophysics: Gas Dynamics and Particle Methods, edit ed by W. Benz, J. Barnes, E. Muller, and M. Norman (Springer-Verlag, New York, 1994), to appear.

[15] D. Bernstein, D. Hobill, E. Seidel, L. Smarr, and J. Towns, Physical Review D (1994), in press.

[16] A. Abrahams, D. Bernstein, D. Hobill, E. Seidel, and L. Smarr, Physical Review D 45, 3544 (1992).

[17] R. Arnowitt, S. Deser, and C. W. Misner, in Gravitation: An Introduction to Current Research, edited by L. Witten (John Wiley, New York, 1962).

[18] C. Misner, Phys. Rev. 118, 1110 (1960).

[19] S. W. Hawking, in Black Holes, edited by C. DeWitt and B. S. DeWitt (Gordon and Breach, New York, 1973).

[20] E. Seidel and W.-M. Suen, Phys. Rev. Lett. 69, 1845 (1992).

[21] P. Anninos, D. Bernstein, S. Brandt, J. Libson, J. Massó, E. Seidel, L. Smarr, W.-M. Suen, and P. Walker, Physical Review Letters (1994), submitted.

[22] E. Leaver, Proc. R. Soc.London A402, 285 (1985).

[23] E. Seidel and S. Iyer, Physical Review D 41, 374 (1990).

[24] E. Newman and R. Penrose, J. Math. Phys. 3, 566 (1962).

[25] E. Newman and T. Unti, J. Math. Phys. 3, 891 (1962).

[26] V. D. Zakharov, Gravitational Waves in Einstein's Theory (Halsted Press, New York, 1973).

[27] D. Bernstein, Ph.D. thesis, Dept. Of Physics, University of Illinois Urbana-Champaign, 1993.

[28] M. Davis, R. Ruffini, H. Press, and R. H. Price, Phys. Rev. Lett. 27, 1466 (1971).

[29] F. J. Zerilli, Phys. Rev. D. 2, 2141 (1970).

[30] M. Davis, R. Ruffini, and J. Tiomno, Physical Review D 5, 2932 (1972).

[31] S. Detweiler and E. Szedenits, Astrophys. J 231, 211 (1979).

[32] K.-I. Oohara and T. Nakamura, Phys. Lett. 94A, 349 (1982).

[33] L. D. Landau and E. M. Lifshitz, The Classical Theory of Fields (Pergamon Press, Oxford, 1975).

[34] Black Holes: The Membrane Paradigm, edited by K. S. Thorne, R. H. Price, and D. A. Macdonald (Yale University Press, London, 1986).

[35] W. M. Suen, R. H. Price, and I. Redmount, Physical Review D 37, 2761 (1988).

[36] R. H. Price and K. S. Thorne, Physical Review D 33, 915 (1986).

[37] M. P. Haugan, S. T. Shapiro, and I. Wasserman, Ap. J. 257, 283 (1982).

[38] R. H. Price and J. Pullin, Physical Review Letters (1994), submitted.

[39] P. Anninos, K. Camarda, J. Massó, E. Seidel, and J. Towns, Physical Review D (1994), in preparation. 
FIG. 1. A single quadrant of the Cadez grid is displayed for the case $\mu=2.2$. The throats are centered on the axis at $z= \pm \operatorname{coth} \mu$. Lines of constant $\eta$ concentrically surround the throats locally, and become spherical far from the holes.

FIG. 2. The metric function $\hat{\gamma}_{\eta \eta}=A$ for the case $\mu=1.2$ is shown at coordinate time $t=25 M$, where $M$ is the mass parameter defined to be half the ADM mass. This configuration was surrounded by a global apparent horizon initially.

FIG. 3. The lapse function $\alpha$ is shown for the case $\mu=1.2$ at the time $t=25 M$. It collapses to zero uniformly around the entire region surrounding both throats, indicating that the system is behaving as a single black hole.

FIG. 4. The lapse function $\alpha$ is shown for the case $\mu=3.25$ at time $t=22.5 M$. At this point the two black holes are still evolving independently of each other.

FIG. 5. The mass of the apparent horizon is shown for the cases $\mu=1.2$ (solid line) and $\mu=2.2$ (dashed line). In the $\mu=1.2$ case the initial horizon surrounds both holes and most of the mass-energy is contained within the horizon. After a slight increase early in the evolution (within the first few $M$ ) the mass of the horizon remains essentially constant until $t \sim 30 M$ when it begins to grow due to numerical effects discussed in the text. In the $\mu=2.2$ case the initial horizons around each throat are distinct. At $t \sim 17 M$ a new apparent horizon appears, surrounding both holes and accounting for essentially all the mass-energy in the system.

FIG. 6. The area of the constant $\eta$ surface that just encircles the saddle point is plotted as a function of $\mu$. The solid line is $16 \pi$, the area corresponding to the total ADM mass of the spacetime (we work with units such that the total mass is normalized to unity).

FIG. 7. We plot various timings of the evolution for the six initial data sets. $t_{n e w t}$ is the Newtonian free fall time required for two particles to collide from rest at this separation, $t_{\text {hor }}$ is the time at which the apparent horizons merged, and $t_{\text {ring }}$ is the time at which the first (negative) peak is seen in the $\ell=2$ Zerilli function recorded at $r=40 M$.

FIG. 8. The $\ell=2$ waveforms for the case $\mu=1.2$. The solid line is the numerically generated waveform extracted at $r=40 M$. The dashed line is a fit of the two lowest $\ell=2$ quasinormal modes, over the domain $70<t / M<160$, to the extracted waveform.

FIG. 9. The $\ell=2$ waveforms for the case $\mu=2.2$. The solid line is the waveform extracted at $r=40 M$ and the long dashed line is the waveform at $r=60 \mathrm{M}$. The dotted line is the quasinormal mode fit.

FIG. 10. The $\ell=4$ waveforms for the case $\mu=2.2$. The solid line is the waveform extracted at $r=40 M$ and the short dashed line is the quasinormal mode fit.

FIG. 11. The $\ell=2$ waveforms for the case $\mu=2.7$. The solid line is the waveform extracted at $r=40 M$ and the short dashed line is the quasinormal mode fit.

FIG. 12. The $\ell=4$ waveforms for the case $\mu=2.7$. The solid line is the waveform extracted at $r=40 M$ and the short dashed line is the quasinormal mode fit.

FIG. 13. The time evolutions for three different radiation indicators are shown for the case $\mu=2.2$. The solid line is the Zerilli function $(\ell=2)$, the dashed line is the Newman-Penrose scalar $\Psi_{4}$, and the dotted line is the radial component of the Bel-Robinson vector. The data tracks the behavior in time of a single point on the grid located at $r=70 M$ along the equator. The $\ell=2$ fundamental quasinormal mode is clearly present and dominant in all three signals. 
FIG. 14. The total gravitational wave energy output is shown for the six parameter studies. The connected circles are the upper limit based on the area theorem, the clustered symbols show numerical results at various detector locations, and the crosses show early results by Smarr and Eppley with their approximate error bars. The solid line labelled "DRPP Calculation" is the result of a naive application of the point particle result, the solid line labelled "Reduced Mass Correction", takes into account the finite mass of the infalling black hole, and the dashed line is the semi-analytic estimate, including several effects discussed in the text. Finally, the dot-dashed line is an empirical fit to the data for low values of the separation parameter $\mu$, showing the exponential falloff of the energy in the one black hole regime.

FIG. 15. We plot the energy factors $F_{r_{o}}$ and $F_{h}$ vs. $r_{o}$. The horizontal axis is the initial Schwarzschild coordinate $r_{o}$ of the infalling hole in units of $M . F_{r_{o}}$ is the gravitational wave energy output for infalling from $r_{o}$ divided by that from $\infty$. $F_{r_{o}}$ is smaller for small $r_{o}$ mainly because the velocity of the infall is smaller. $F_{h}$ is the percentage of the available energy compared to the total gravitational energy available after taking into account the dissipation due to tidal distortions of the black hole horizons. As $r_{o} \rightarrow \infty, F_{h}$ tends to $86 \%$. The curves are calculated for $m=M$.

FIG. 16. Development of horizon shear and expansion. The horizontal axis is the Schwarzschild coordinate $r$ of the infalling object in units of $M$. The solid line represents the magnitude of the horizon shear on the horizon of the infalling hole $m$. The dotted line is the expansion. Both the shear and the expansion are in units of $m^{-1}$ and are plotted for the case $m=M$.

FIG. 17. The reabsorption of the $l=2$ wave. The horizontal axis is the angular frequency $\omega$ of the $l=2$ wave in units of $M^{-1}$. The dashed line is $d E / d \omega$, the dotted line is the transmission coefficient of the $l=2$ wave multiplied by 0.1 and the solid line is the product $T(d E / d \omega)$. For $M=m$ the area under the solid curve, which is found to be $0.0012 m$, is roughly the total reabsorption of the $l=2$ wave by the infalling hole.

\begin{tabular}{|c|r|r|r|r}
\hline \hline \hline$\mu$ & $M$ & $L / M$ & Apparent horizon & Event horizon \\
\hline 1.2 & 1.85 & 4.46 & global & global \\
\hline 1.8 & 0.81 & 6.76 & separate & critical \\
\hline 2.2 & 0.50 & 8.92 & separate & separate \\
\hline 2.7 & 0.29 & 12.7 & separate & separate \\
\hline 3.0 & 0.21 & 15.8 & separate & separate \\
\hline 3.25 & 0.16 & 19.1 & separate & separate \\
\hline \hline
\end{tabular}

TABLE I. The physical parameters for the six initial data sets. $M$ is the mass parameter equal to half the ADM mass of the spacetime, $L / M$ is the proper distance between the throats, and we note whether or not a single apparent or event horizon surrounds both holes.

\begin{tabular}{|c|c|c|r|}
\hline \hline$\mu$ & $E_{\psi}$ & $E_{\Psi_{4}}$ & $E_{p \gamma}$ \\
\hline 1.2 & $1.34-0.99 \times 10^{-5}$ & $9.81-1.32 \times 10^{-5}$ & $7.58-1.29 \times 10^{-5}$ \\
\hline 1.8 & $1.95-1.64 \times 10^{-4}$ & $3.46-1.72 \times 10^{-4}$ & $3.77-1.73 \times 10^{-4}$ \\
\hline 2.2 & $6.10-5.27 \times 10^{-4}$ & $7.76-5.21 \times 10^{-4}$ & $8.91-5.26 \times 10^{-4}$ \\
\hline 2.7 & $7.50-6.86 \times 10^{-4}$ & $7.16-5.00 \times 10^{-4}$ & $7.52-5.07 \times 10^{-4}$ \\
\hline 3.0 & $8.85-7.13 \times 10^{-4}$ & $9.22-4.86 \times 10^{-4}$ & $10.0-4.93 \times 10^{-4}$ \\
\hline 3.25 & $1.37-0.85 \times 10^{-3}$ & $4.32-1.11 \times 10^{-3}$ & $3.39-1.11 \times 10^{-3}$ \\
\hline \hline
\end{tabular}

TABLE II. The total radiated energy for the six initial data sets normalized to the ADM mass (2M) of the spacetime. We compare results using several different methods of calculation on a grid resolved with $200 \times 35$ zones. The energies are extracted at five different radii, and the range given here is from the innermost to the outermost radius. See text for details. 